\title{
Mitochondrial complex I inhibition triggers a mitophagy-dependent ROS increase leading to necroptosis and ferroptosis in melanoma cells
}

\author{
Farhan Basit ${ }^{1,4}$, Lisanne MPE van Oppen ${ }^{1,4}$, Laura Schöckel ${ }^{2}$, Hasse M Bossenbroek ${ }^{1}$, Sjenet E van Emst-de Vries ${ }^{1}$, \\ Johannes CW Hermeling ${ }^{1}$, Sander Grefte ${ }^{1,5}$, Charlotte Kopitz ${ }^{2}$, Melanie Heroult ${ }^{3}$, Peter HGM Willems ${ }^{1}$ and Werner JH Koopman ${ }^{*, 1}$
}

\begin{abstract}
Inhibition of complex I (CI) of the mitochondrial respiratory chain by BAY 87-2243 ('BAY') triggers death of BRAF ${ }^{\mathrm{V} 600 \mathrm{E}}$ melanoma cell lines and inhibits in vivo tumor growth. Here we studied the mechanism by which this inhibition induces melanoma cell death. BAY treatment depolarized the mitochondrial membrane potential $(\Delta \psi)$, increased cellular ROS levels, stimulated lipid peroxidation and reduced glutathione levels. These effects were paralleled by increased opening of the mitochondrial permeability transition pore (mPTP) and stimulation of autophagosome formation and mitophagy. BAY-induced cell death was not due to glucose shortage and inhibited by the antioxidant $\alpha$-tocopherol and the MPTP inhibitor cyclosporin A. Tumor necrosis factor receptor-associated protein 1 (TRAP1) overexpression in BAY-treated cells lowered ROS levels and inhibited mPTP opening and cell death, whereas the latter was potentiated by TRAP1 knockdown. Knockdown of autophagy-related 5 (ATG5) inhibited the BAY-stimulated autophagosome formation, cellular ROS increase and cell death. Knockdown of phosphatase and tensin homologinduced putative kinase 1 (PINK1) inhibited the BAY-induced $\Delta \psi$ depolarization, mitophagy stimulation, ROS increase and cell death. Dynamin-related protein 1 (Drp1) knockdown induced mitochondrial filamentation and inhibited BAY-induced cell death. The latter was insensitive to the pancaspase inhibitor z-VAD-FMK, but reduced by necroptosis inhibitors (necrostatin-1, necrostatin-1s)) and knockdown of key necroptosis proteins (receptor-interacting serine/threonine-protein kinase 1 (RIPK1) and mixed lineage kinase domain-like (MLKL)). BAY-induced cell death was also reduced by the ferroptosis inhibitor ferrostatin-1 and overexpression of the ferroptosis-inhibiting protein glutathione peroxidase 4 (GPX4). This overexpression also inhibited the BAYinduced ROS increase and lipid peroxidation. Conversely, GPX4 knockdown potentiated BAY-induced cell death. We propose a chain of events in which: (i) $\mathrm{Cl}$ inhibition induces mPTP opening and $\Delta \psi$ depolarization, that (ii) stimulate autophagosome formation, mitophagy and an associated ROS increase, leading to (iii) activation of combined necroptotic/ferroptotic cell death. Cell Death and Disease (2017) 8, e2716; doi:10.1038/cddis.2017.133; published online 30 March 2017
\end{abstract}

To sustain their function and proliferation melanoma cells often shift their metabolism from mitochondrial towards glycolytic ATP production. ${ }^{1}$ However, various oncogenes and tumor suppressors (e.g. c-myc, Ras and Oct1), as well as hypoxia, stimulate mitochondrial metabolism. ${ }^{2-5}$ A key oncogenic event in melanoma is the occurrence of mutations in v-Raf murine sarcoma viral oncogene homolog $B$ (BRAF). This protein kinase is involved in RAS-RAF-MEK-ERK mitogen-activated protein kinase signaling. ${ }^{1}$ Among the BRAF mutations, the V600E gain-of-function substitution is most commonly observed (i.e. in $40-60 \%$ of all melanomas). In addition, BRAF mutations were demonstrated in $40 \%$ of the thyroid cancers, $30 \%$ of the ovarian cancers and $20 \%$ of the colorectal cancers. $^{6,7}$ Despite novel antitumour therapeutics, metastatic melanoma still has a poor prognosis due to the development of chemotherapy resistance. ${ }^{8}$ Importantly, acquired resistance to BRAF or MEK inhibitors was paralleled by increased mitochondrial biogenesis and activity in melanoma cells with BRAF $^{\mathrm{V} 600 \mathrm{E}}$ and NRAS mutations. ${ }^{9,10}$ This suggests that concomitant inhibition of mitochondrial function might constitute a potential therapeutic strategy. ${ }^{11,12}$ Proper mitochondrial functioning requires activity of the mitochondrial oxidative phosphorylation (OXPHOS) system. ${ }^{13-15}$ This system is embedded in the mitochondrial inner membrane (MIM) and consists of four electron transport chain (ETC) complexes $(\mathrm{Cl}-\mathrm{CIV})$ and the $\mathrm{F}_{0} \mathrm{~F}_{1}$-ATP-synthase (CV). OXPHOS generates ATP through chemiosmotic coupling by linking ETC-mediated proton efflux across the MIM to CV-mediated trans-MIM proton influx. ${ }^{16}$ The latter is driven by the inwarddirected proton motive force across the MIM, which consists of an electrical $(\Delta \psi)$ and chemical $(\Delta \mathrm{pH})$ component, with $\Delta \psi$ contributing $\sim 85 \%$ to the total PMF. ${ }^{17}$

Using a panel of BRAF ${ }^{\mathrm{V} 600 E}$ melanoma cell lines, we recently demonstrated that BAY 87-2243 (BAY; Ellinghaus et $\mathrm{al}^{18}$ ), a potent inhibitor of the first OXPHOS complex (Cl or NADH:ubiquinone oxidoreductase; EC 1.6.5.3), dose-dependently decreases cell viability. ${ }^{19}$ The effect of BAY was paralleled by increased levels of cellular ROS and a

\footnotetext{
${ }^{1}$ Department of Biochemistry, Radboud Institute for Molecular Life Sciences (RIMLS), Radboud University Medical Centre (Radboudumc), Nijmegen, The Netherlands; ${ }^{2}$ Global Therapeutic Research Group Oncology II, Bayer Pharma AG, Berlin, Germany and ${ }^{3}$ Bayer AG Innovation Strategy, Leverkusen, Germany

${ }^{*}$ Corresponding author: WJH Koopman, Department of Biochemistry (286), Radboud Institute for Molecular Life Sciences (RIMLS), Radboud University Medical Centre (Radboudumc), PO Box 9101, 6500 HB Nijmegen, The Netherlands. Tel: +31 24 3614589; Fax: +31 24 3616413; E-mail: Werner.Koopman@radboudumc.nl

${ }^{4}$ These authors contributed equally to this work.

${ }^{5}$ Current address: Department of Human and Animal Physiology, Wageningen University and Research, Wageningen, The Netherlands.

Received 24.10.16; revised 23.2.17; accepted 28.2.17; Edited by E Baehrecke
} 
reduction in cellular ATP levels. Measurements were performed using a medium with a physiological glucose concentration $(5 \mathrm{mM})$ and cell death was prevented by increasing this concentration to $25 \mathrm{mM}$. Remarkably, using the latter concentration also inhibited the BAY-induced ROS increase and reduction in ATP level. ${ }^{19}$ Mechanistically, we hypothesized that BAY-induced $\mathrm{Cl}$ inhibition promotes cell death by stimulating ATP-depletion and/or ROS generation and subsequent oxidative stress induction. Here we dissected the mechanism by which BAY kills BRAF ${ }^{\mathrm{V} 600 \mathrm{E}}$ melanoma cells. Our results suggest that this death is not due to glucose shortage but involves a chain of events by which BAY stimulates opening of the mitochondrial permeability transition pore (mPTP) and $\Delta \psi$ depolarization, followed by autophagosome formation, mitophagy, a cytosolic ROS increase and combined necroptosis/ferroptosis.

\section{Results}

BAY treatment induces cell death in BRAF ${ }^{\mathrm{V} 600 \mathrm{E}}$ melanoma cell lines. In this study, we used two BRAF ${ }^{\mathrm{V} 600 \mathrm{E}}$ melanoma cell lines (G361 and SK-MEL-28) to investigate the mechanism of BAY-induced cell death. We previously demonstrated $^{19}$ that BAY treatment for $72 \mathrm{~h}$ reduced the viability of these cells in a dose-dependent manner with $\mathrm{IC}_{50}$ values in the nanomolar range (Figure 1a). Within this timeframe, BAY did not affect the viability of human epidermal melanocytes (Hema-LP) and primary human skin fibroblasts (CT5120; Supplementary Figure S1A). Experiments were performed at an ambient glucose concentration of $5 \mathrm{mM}$. Importantly, regular refreshment of the culture medium did not prevent the BAY-induced reduction in cell viability, arguing against glucose depletion being responsible for this reduction (Supplementary Figure S1B). In agreement with our previous study, ${ }^{19}$ it was found that BAY displayed a half-maximal inhibition of cell viability $\left(T_{1 / 2}\right)$ at 20 and $66 \mathrm{~h}$ for $\mathrm{G} 361$ and SK-MEL-28 cells, respectively (Figure $1 \mathrm{~b}$ ). This means that
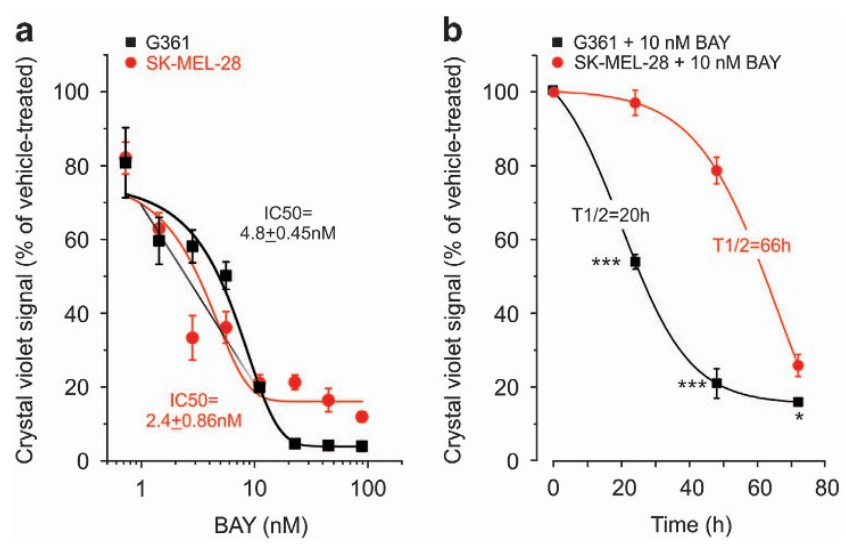

Figure 1 Dose- and time-dependent effect of BAY and medium refreshment on cell viability. (a) Dose-dependent effect of BAY $(N=2, n=4)$ on the viability of $G 361$ and SK-MEL-28 cells (at $72 \mathrm{~h}$ ). A Boltzmann equation was used to determine the $\mathrm{IC}_{50}\left(x_{0}\right)$ value: $y=\left(A_{2}+\left(A_{1}-A_{2}\right) /\left(1+\exp \left(\left(x-x_{0}\right) / \mathrm{d} x\right)\right)\right)$. (b) G361 and SK-MEL-28 melanoma cells were treated with $10 \mathrm{nM} \mathrm{BAY}(N=3, n=6)$ and their viability was measured at different time points. A sigmoidal (logistic) equation was used to determine the $T_{1 / 2}\left(x_{0}\right)$ value: $y=\left(\left[\left(A_{1}-A_{2}\right) /\left(1+\left(x / x_{0}\right)\right]+A_{2}\right)\right.$. Statistics: In panel $b$, significant differences with the SK-MEL-28 cell line are indicated by ${ }^{*} P<0.05$ and ${ }^{* * *} P<0.001$
G361 cells are more sensitive to $\mathrm{Cl}$ inhibition than SKMEL-28 cells. ${ }^{19}$ Therefore, to study cell death an incubation time was chosen at which a similar reduction in viability for G361 (48 h) and SK-MEL-28 (72 h) cells was observed. Under these conditions, BAY treatment induced cell death to the same extent $(70 \%)$ in both cell lines (e.g. Figure $2 \mathrm{~d}$ ).

TRAP1 overexpression inhibits BAY-stimulated mPTP opening, cellular ROS increase and cell death. Activation of BRAF signalling leads to increased phosphorylation of tumor necrosis factor receptor-associated protein 1 (TRAP1), which displays antiapoptotic properties in cancer cells. ${ }^{20}$ TRAP1 is also involved in the regulation of mPTP opening. ${ }^{21}$ To assess whether TRAP1 and MPTP opening play a role in BAY-induced cell death, we used a previously described method to measure MPTP opening. ${ }^{22}$ In brief, cells are stained with the fluorescent cation tetramethylrhodamine methyl ester (TMRM), which accumulates in the mitochondrial matrix in a $\Delta \psi$-dependent manner. ${ }^{23}$ Next, reversible mPTP openings are photoinduced by controlled illumination of TMRM (Supplementary Figure S2 and Supplementary Movie). For quantification, individual mPTP openings were manually counted from 'difference images' calculated by subtracting the $(n-1)^{\text {th }}$ image from the $n^{\text {th }}$ image in the recorded TMRM image sequence (Figure 2a; e.g. img422img421). In these difference images individual mPTP openings are highlighted in black (Figure 2a; arrowhead). Acute BAY treatment $(2 \mathrm{~min})$ increased the number of $\mathrm{mPTP}$ openings to a similar extent in both cell lines (Figure $2 b$ ). Of note, given the short incubation time we here used a $40 \mathrm{nM}$ BAY concentration. This was the lowest BAY concentration that maximally inhibited oxygen consumption in G361 and SK-MEL-28 cells during acute treatment. ${ }^{19}$ The acute effect of BAY on mPTP opening (Figure $2 b$ ) and its chronic effect on cell death (Figure $2 \mathrm{~d}$ ) were inhibited by pre-treatment $(2 \mathrm{~h})$ with the mPTP inhibitor cyclosporin A (CsA). TRAP1 overexpression (Supplementary Figure S3A) mimicked both effects of CsA (Figures $2 b-e$ ). These results suggest that TRAP1 inhibits MPTP opening to prevent cell death in BRAF $^{\mathrm{V} 600 \mathrm{E}}$ melanoma cells and that TRAP1 overexpression is required to inhibit BAY-induced $\mathrm{MPTP}$ opening and cell death. In agreement with this idea, TRAP1 knockdown (Supplementary Figure S3A) increased the sensitivity to BAY-induced cell death as compared with SiCTRL (Figure 2e). TRAP1 overexpression significantly reduced the increase in cellular ROS levels after $24 \mathrm{~h}$ of BAY treatment (Figure 2c). Taken together, these results suggest that stimulation of mPTP opening is a key component of BAYinduced cell death and that endogenous TRAP1 levels and/or TRAP1 activity are too low to protect against this death. The fact that TRAP1 overexpression lowers ROS levels in BAYtreated cells suggests that TRAP1 directly reduces these levels or that MPTP opening induces ROS.

ATG5 knockdown inhibits BAY-stimulated autophagosome formation, ROS increase and cell death. Elevated ROS levels and increased MPTP opening are established stimulators of autophagy. ${ }^{24}$ To test the hypothesis that BAY stimulates autophagosome formation, cells were transfected with the biosensor mCherry-GFP-tagged LC3. Quantification 


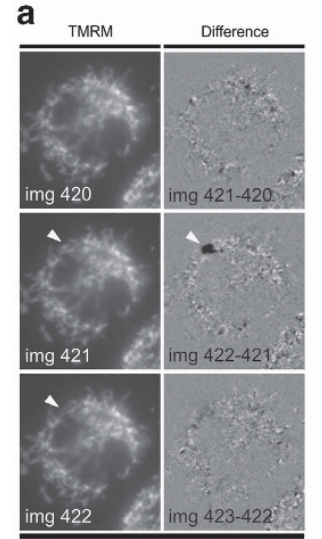

G361: Empty vector

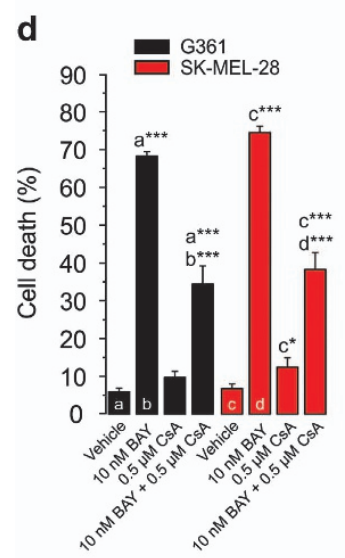

b

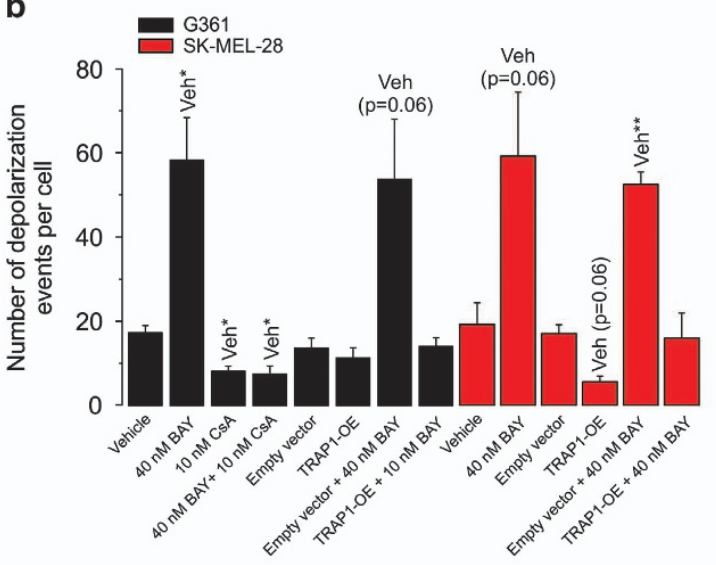

C

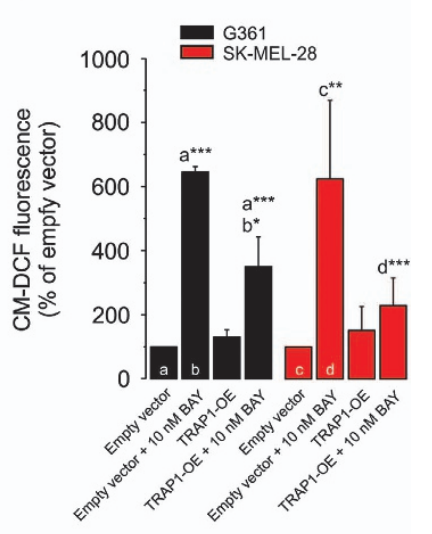

e

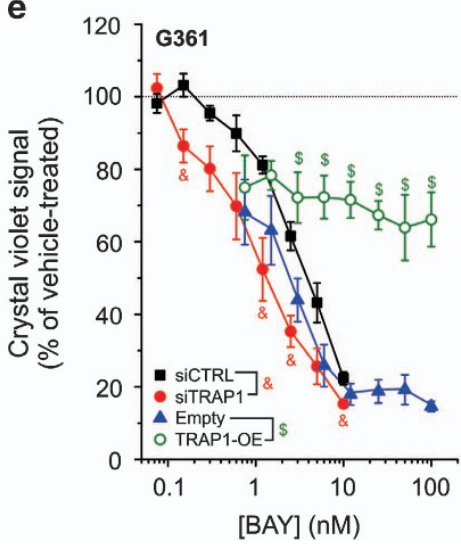

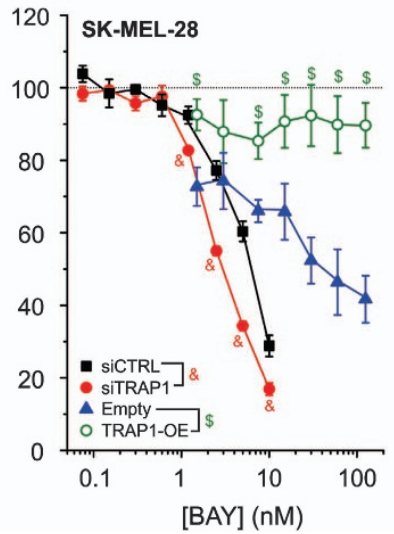

Figure 2 Effect of TRAP1 knockdown/overexpression on the BAY-induced reduction in cell viability. (a) Detection of photoinduced mitochondrial membrane potential $(\Delta \psi)$ depolarizations (' $\Delta \psi$-flickering') in a typical TMRM-stained G361 cells (see Results for details). (b) Effect of BAY (2 min), CsA (2 h), empty vector and TRAP1-OE on $\Delta \psi$ flickering $(N=3, n \geq 20)$. (c) Effect of BAY on reactive oxygen species (ROS) levels (at $24 \mathrm{~h} ; \mathrm{N}=3, n=3$ ) in cells transfected with the empty or TRAP1-OE vector. (d) Effect of vehicle $(N=5, n=15)$, BAY $(N=5, n=15)$ and CsA $(N=3, n=6)$ on cell death (G361: at $48 \mathrm{~h}$; SK-MEL-28: at $72 \mathrm{~h})$. (e) Effect of BAY on the viability of cells (G361: at 48 h; SK-MEL-28: at $72 \mathrm{~h} ; N=3, n=6$ ) transfected with siCTRL, siTRAP1, empty vector and TRAP1-OE. Statistics: Significant differences relative to the vehicle condition ('Veh' in panel b) and between treatments $(a, b, c, d$ in panels $\mathbf{c}$ and $\mathbf{d})$ are indicated by ${ }^{*} P<0.05,{ }^{* *} P<0.01$ and ${ }^{* * *} P<0.001$. In panel (e), significant differences $(P<0.05)$ between conditions are marked by symbols $(\&, \$)$

of the number of green GFP puncta per cell, representing autophagosomes, revealed a significant BAY-induced increase (Supplementary Figure S4A and B and Figure 3a). Co-incubation with bafilomycin A1 (BafA1), which prevents fusion of autophagosomes with lysosomes, ${ }^{25}$ further increased the number of green GFP puncta. This suggests that BAY does not inhibit autophagosome fusion but stimulates autophagosome formation. We previously demonstrated that the antioxidant $a$-tocopherol (TOC) inhibits the BAY-induced increase in cellular ROS levels and cell death. ${ }^{19}$ Here we observed that $24 \mathrm{~h}$ cotreatment with TOC fully prevents BAY-induced stimulation of autophagosome formation (Figure 3a). To further investigate the role of autophagosome formation in BAY-induced cell death, we performed knockdown of autophagy-related 5 (ATG5; Supplementary Figure S3B). This protein is required for autophagosome formation ${ }^{26}$ and its knockdown significantly reduced the BAYinduced increase in green puncta (Figure 3a). Remarkably, ATG5 knockdown also prevented the ROS increase after $24 \mathrm{~h}$ BAY treatment (Figure $3 b$ ). Analysis of cellular mitochondrial content using Mitotracker Green FM (MG) revealed that BAY treatment reduced MG staining and that this reduction was prevented by ATG5 knockdown (Figure 3c). This suggests that BAY-induced stimulation of ATG5-dependent autophagosome formation mediates mitochondria-specific autophagy (i.e. mitophagy). ATG5 knockdown inhibited BAY-induced loss of cell viability (Figure 3d). Taken together, these data suggest that TOC-sensitive 'triggering ROS' is required for mPTP opening and subsequent ATG5-mediated autophagosome formation. Moreover, our results suggest that ATG5-mediated autophagosome formation is required for sustained elevated ROS and increased mitophagy and eventually BAY-induced cell death.

PINK1 knockdown inhibits BAY-stimulated mitophagy, $\Delta \psi$ depolarization, ROS increase and cell death. To demonstrate the potential involvement of mitophagy in BAYinduced cell death, cells were transfected with GFP-LC3 (marking autophagosomes) and stained with MitoTracker Red (MR) to highlight mitochondria. Then, the number of green GFP puncta colocalizing with MR was determined to quantify the amount of mitophagy (Supplementary Figure S4C; 
a

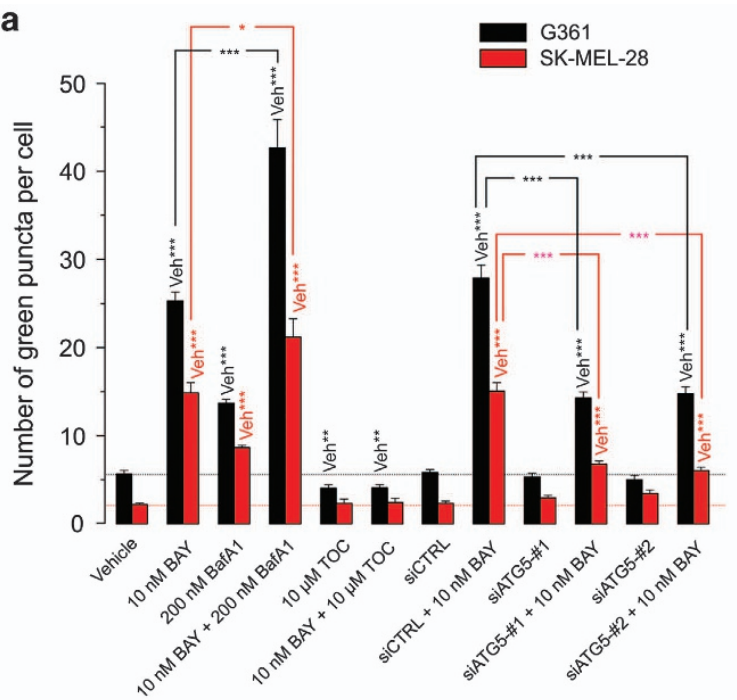

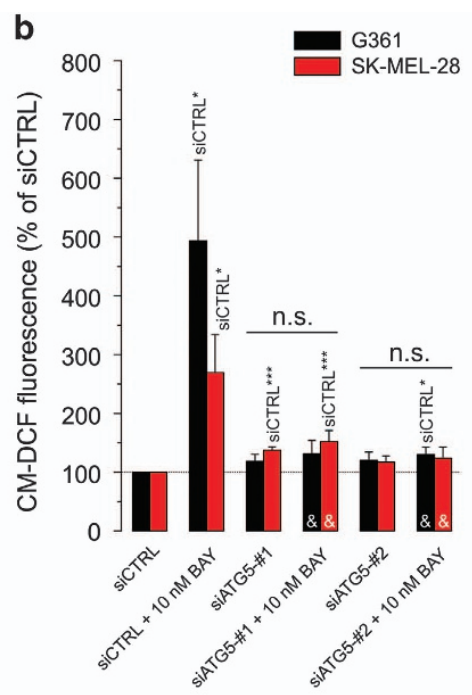
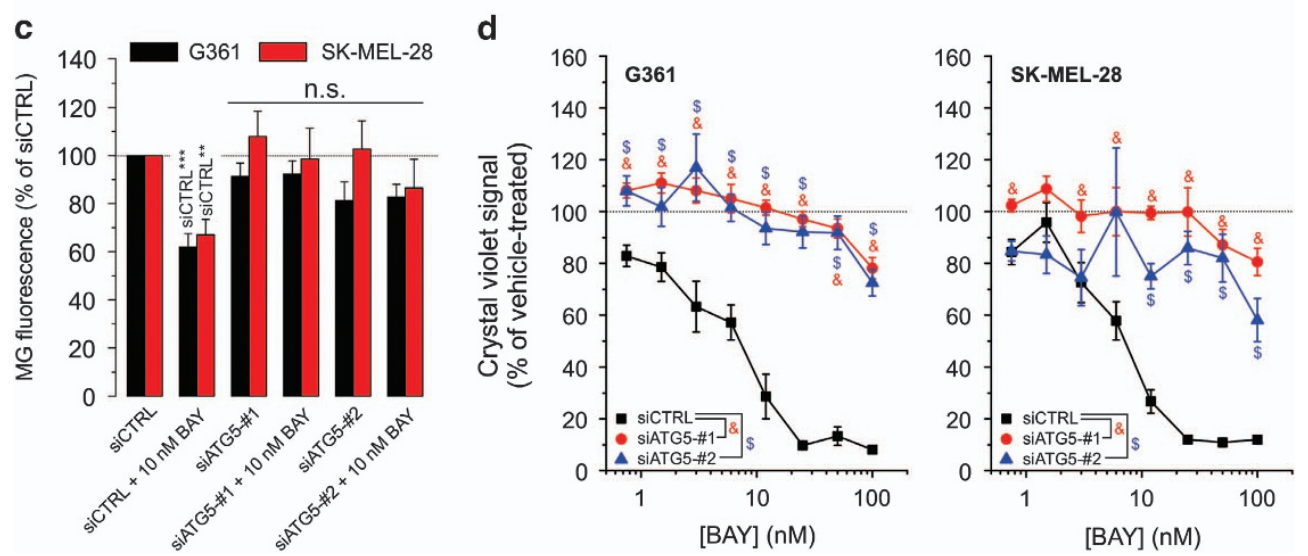

Figure 3 Effect of ATG5 knockdown on the BAY-induced stimulation of autophagy, reactive oxygen species (ROS) increase and reduction in cell viability. (a) Effect of BAY in the absence and presence of BafA1, TOC and ATG5 knockdown on the number of green puncta in G361 and SK-MEL-28 cells (at 24 h; N=3, $n=30$ ). (b) Effect of BAY on ROS levels (at $24 \mathrm{~h} ; N=3, n=9$ ) in cells transfected with an empty or GPX4 overexpression vector (GPX-OE). (c) Effect of BAY on cellular MG fluorescence (G361: at $24 \mathrm{~h}$; SK-MEL-28: at $24 \mathrm{~h} ; N=3, n=9$ ) in cells transfected with siCTRL, siATG5-no. 1 and siATG5-no. 2. (d) Effect of BAY on the viability of cells (G361: at 48 h; SK-MEL-28: at $72 \mathrm{~h}$; $N=3, n=6$ ) transfected with siCTRL, siATG5-no. 1 and siATG5-no. 2. Statistics: Significant differences relative to the marked conditions are indicated by ${ }^{\star} P<0.05,{ }^{* *} P<0.01$ and ${ }^{* * *} P<0.001$. NS indicates nonsignificant. In panel (d), significant differences with the (siCTRL+10 nM BAY condition) are marked by ' $\&$ '. In panel (b) significant differences $(P<0.05)$ between conditions are marked by symbols $(\&, \$)$

arrowheads). BAY treatment $(24 \mathrm{~h})$ stimulated mitophagy (Figure $4 \mathrm{a}$ ) and induced $\Delta \psi$ depolarization (Figure 4b). Phosphatase and tensin homolog-induced putative kinase 1 (PINK1) is a key regulator of mitophagy that recruits autophagy receptors to mitochondria upon $\Delta \psi$ depolarization. ${ }^{27}$ PINK1 knockdown (Supplementary Figure S3C) inhibited the BAY-induced changes (Figures $4 a-d$ ) as well as the BAY-induced reduction in cell viability (Figure $4 \mathrm{e}$ ). The extent of mitophagy induction was positively correlated with the degree of $\Delta \psi$ depolarization (Supplementary Figure S1C), compatible with the key role of $\Delta \psi$ depolarization in mitophagy induction. ${ }^{28}$ These results suggest that BAY induces $\Delta \psi$ depolarization, thereby stimulating PINK1-dependent mitophagy and an ensuing increase in cellular ROS levels.

Drp1 knockdown induces mitochondrial filamentation and inhibits BAY-stimulated cell death. In a starvation model, ${ }^{29}$ autophagosomal degradation of mitochondria was prevented by mitochondrial elongation (i.e. filamentation) mediated by downregulation of dynamin-related protein 1 (Drp1). This GTPase is one of the key mediators of mitochondrial fission. ${ }^{30}$ Two Drp1 isoforms, the brain ('b') and ubiquitous ('u') form, were detected in G361 and SK-MEL-28 cells. Knockdown of both isoforms (Supplementary Figure S3D) induced mitochondrial filamentation, both in the absence and presence of BAY (Figures $5 a$ and b). Drp1 knockdown also inhibited the BAY-induced reduction in cell viability (Figure $5 \mathrm{c}$ ). Compatible with these results (Supplementary Figure S1D), BAY-induced cell death was inhibited by a chemical inhibitor of Drp1 activity (Mdivi1; Cassidy-Stone et $a l^{31}{ }^{1}$ ). Mdivi1 also inhibited the BAY-induced stimulation of mitophagy (Supplementary Figure S1E). These results suggest that BAY-induced mitophagy stimulation and cell killing are inhibited by mitochondrial filamentation. 

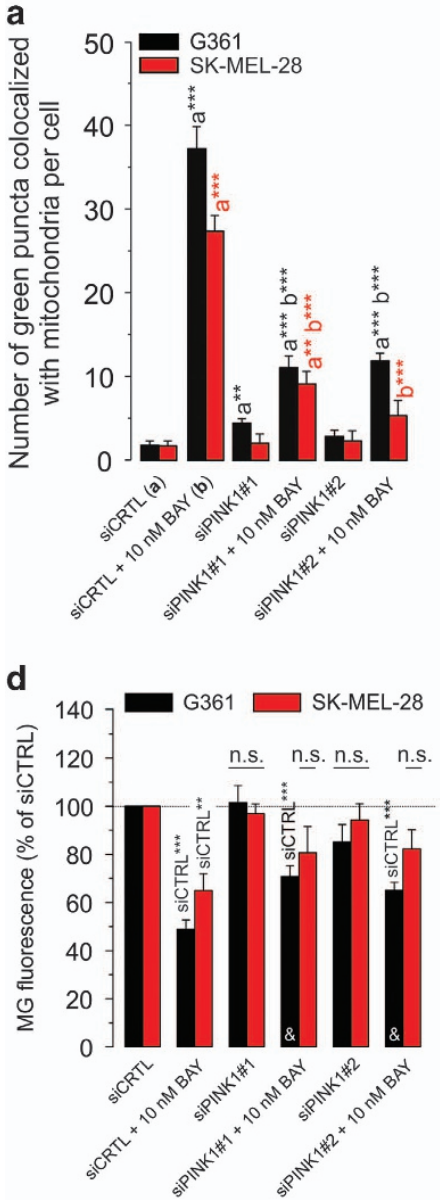
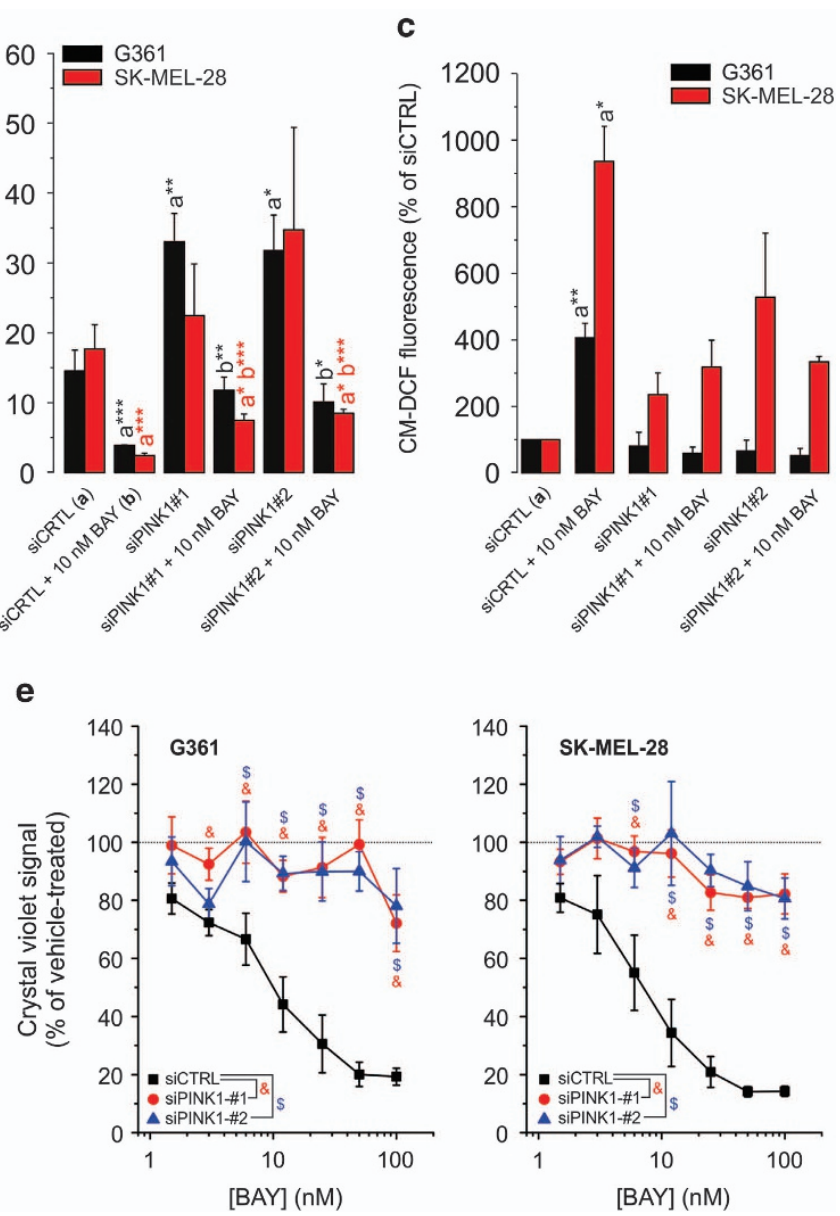

Figure 4 Effect of PINK1 knockdown on the BAY-induced stimulation of mitophagy, $\Delta \psi$ depolarization, reactive oxygen species (ROS) levels and reduction in cell viability. (a) Effect of BAY treatment, siPINK1-no. 1 and siPINK1-no. 2 on the number of green puncta colocalizing with mitochondria in G361 and SK-MEL-28 cells (at $24 \mathrm{~h} ; N=3, n \geq 14$ ). (b) Similar to panel a, but now for the effect on mitochondrial membrane potential (i.e. the JC-1 red/green ratio signal; $N=3, n=6$ ). (c) Similar to panel a, but now for the effect on cellular ROS levels $(N=3, n=6$ ). (d) Similar to panel a, but now for the effect on cellular MG fluorescence intensity (G361: at $24 \mathrm{~h} ; \mathrm{SK}-\mathrm{MEL}-28$ : at $24 \mathrm{~h} ; N=3, n=9$ ). (e) Effect of BAY on the viability of cells (G361: at $48 \mathrm{~h}$; SK-MEL-28: at $72 \mathrm{~h} ; N=3, n=6$ ) transfected with siCTRL, siPINK1-no. 1 and siPINK1-no. 2. Statistics: Significant differences relative to the indicated conditions are marked by ${ }^{*} P<0.05,{ }^{*} P<0.01$ and ${ }^{* * *} P<0.001$. In panel $\mathrm{c}$ statistical analysis was performed using a one-sample t-test against a value of 100. In panel d, NS indicates nonsignificant and significant differences with the (siCTRL+10 nM BAY condition) are marked by ' $\&$ '. In panel (e) significant differences $(P<0.05)$ between conditions are marked by symbols $(\&, \$)$

BAY-induced cell death involves necroptosis and ferroptosis rather than apoptosis. Stimulation of autophagy was linked to induction of both apoptotic and necroptotic cell death. ${ }^{32-36}$ The broad-spectrum caspase inhibitor Z-VAD-FMK was unable to prevent BAY-induced cell death (Figure 6a), arguing against involvement of apoptosis. On the other hand, Nec-1, which blocks necroptosis by inhibiting the activity of receptor-interacting serine/threonine-protein kinase 1 (RIPK1), ${ }^{37,38}$ inhibited BAY-induced cell death (Figures $6 a$ and b). However, Nec-1 not only inhibits RIPK1 but also indoleamine-2,3-dioxygenase, which catalyzes the conversion of tryptophan into kynurenine. ${ }^{39,40}$ Therefore, we next investigated the effect of $\mathrm{Nec}-1 \mathrm{~s}$ (or 7-Cl-O-Nec-1), a RIPK1 inhibitor displaying increased stability and specificity. ${ }^{39,41}$ Nec-1s also inhibited BAY-induced cell death, albeit to a lesser extent than Nec-1 (Figure 6b), suggesting that this death is partially mediated by necroptosis. Compatible with this conclusion, knockdown of RIPK1 inhibited the BAY-induced loss in cell viability (Supplementary Figure S3E and Figure 6c).
However, RIPK1 kinase activity is not only required for necroptosis but also for apoptosis. ${ }^{42}$ For this reason, we performed knockdown of the mixed lineage kinase domain-like protein (MLKL), the presence of which is essential for necroptosis induction. ${ }^{41,43}$ Similar to RIPK1, MLKL knockdown inhibited the cell viability loss in BAY-treated cells (Supplementary Figure S3F and Figure 6d). Nec-1s only partially blocked BAY-induced cell death (Figure 6b), suggesting that necroptosis is not the only death mechanism involved. In this sense, autophagy can also promote ferroptosis, ${ }^{44}$ a mode of cell death that is negatively regulated by glutathione peroxidase 4 (GPX4) and characterized by increased irondependent ROS production, glutathione (GSH) depletion and lipid peroxidation. ${ }^{41}$ Supporting the involvement of ferroptosis, BAY treatment increased cytosolic ROS levels (see above), reduced cellular GSH levels (Figure 7a) and stimulated TOCsensitive lipid peroxidation (Figure 7c). Moreover, we previously demonstrated that cotreatment with the GSH precursor $\mathrm{N}$-acetyl cysteine inhibited the BAY-induced increase in cellular 
a
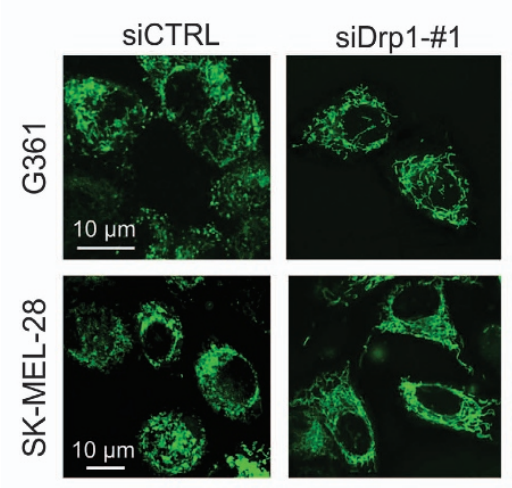

\section{b}

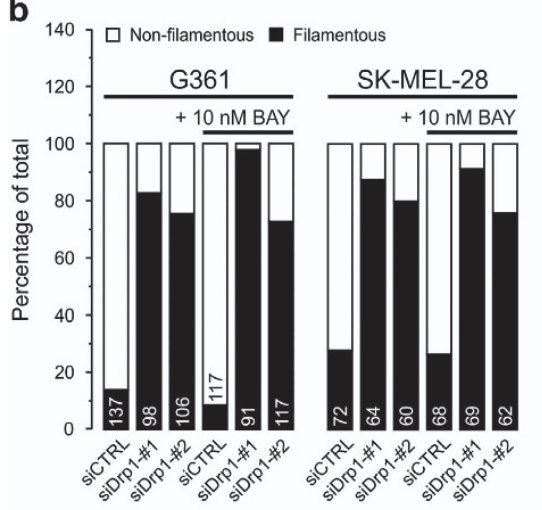

siDrp1-\#1

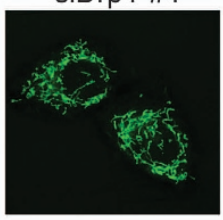

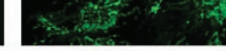

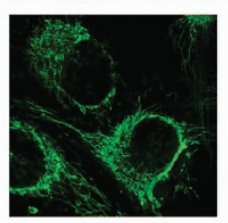

C

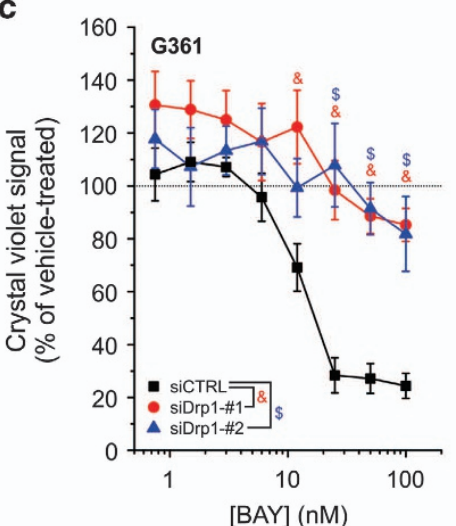

$+10 \mathrm{nM}$ BAY
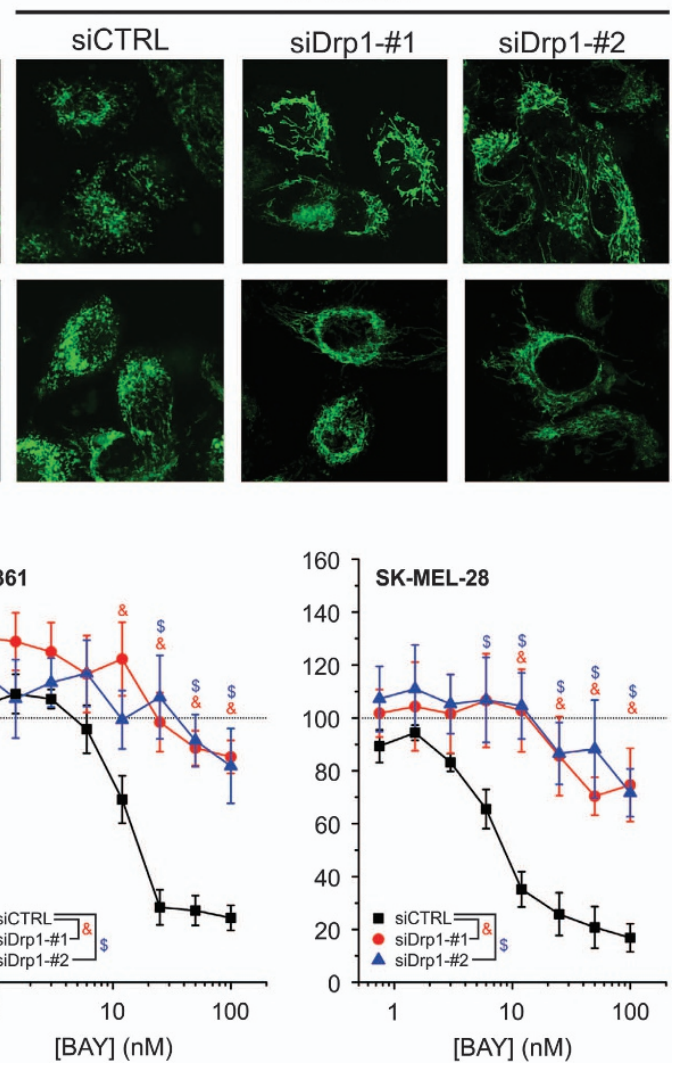

Figure 5 Effect of Drp1 knockdown on mitochondrial morphology and the BAY-induced reduction in cell viability. (a) Typical examples visualizing mitochondria morphology in MG-stained cells treated with siCTRL, siDrp1-no. 1 and siDrp1-no. 2 in the absence and presence of $10 \mathrm{nM} \mathrm{BAY} \mathrm{(G361:} \mathrm{at} \mathrm{16} \mathrm{h;} \mathrm{SK-MEL-28:} \mathrm{at} 24$ h). (b) Quantification of mitochondrial morphology in multiple cells ( $N=2$; numerals indicate the number of cells analyzed) for the conditions in panel (a). (c) Effect of BAY on the viability of cells (G361: at 48 h; SK-MEL-28: at $72 \mathrm{~h} ; N=3, n=6)$ transfected with siCTRL, siDrp1-no. 1 and siDrp1-no. 2. Statistics: In panel (c), statistically significant differences $(P<0.05)$ between conditions are marked by symbols $(\&, \$)$

ROS levels and cell death. ${ }^{19}$ The ferroptosis inhibitor ferrostatin-1 (Fer-1; Dixon et al. ${ }^{45}$ ) partially prevented BAYinduced cell death (Figures $6 a$ and b). Overexpression of the ferroptosis-inhibiting enzyme GPX4 (Supplementary Figure S3G) inhibited the BAY-stimulated increase in cellular ROS levels (Figure 7b) and lipid peroxidation (Figure 7c). In agreement with this result, GPX4 overexpression and knockdown (Supplementary Figure S3G) inhibited and potentiated the BAY-induced reduction in cell viability, respectively (Figure 7d). Overall, these results suggest that BAY treatment does not induce apoptotic cell death but triggers combined necroptosis and ferroptosis.

\section{Discussion}

Here we provide mechanistic insight into how BAY-induced inhibition of mitochondrial $\mathrm{Cl}$ induces death in BRAF $\mathrm{V} 600 \mathrm{E}$ melanoma cells. A chain of events is proposed (Figure 8 ), in which $\mathrm{Cl}$ inhibition stimulates $\mathrm{MPTP}$ opening, $\Delta \psi$ depolarization, autophagosome formation and mitophagy induction. The latter increases cellular ROS levels that stimulate lipid peroxidation and GSH depletion, leading to combined necroptotic and ferroptotic cell death.
Specificity of BAY for melanoma cells. Within the used timeframe, BAY-induced $\mathrm{Cl}$ inhibition effectively killed G361 and SK-MEL-28 melanoma cells, without affecting the viability of non-cancer cells. It was proposed that cancer cells displaying a higher basal ROS level than normal cells can be therapeutically targeted by ROS-inducing anticancer agents. ${ }^{46}$ This suggests that BAY treatment increases ROS levels beyond a death-inducing threshold in cancer cells but not in non-cancer cells. Compatible with this idea, we observed that G361 cells displayed a fourfold higher basal ROS level than SK-ML-28 cells (Supplementary Figure S1F). This might explain why BAY-induced killing occurred at a much earlier time point in G361 cells than in SK-MEL-28 cells.

Role of external glucose. We previously demonstrated in C2C12 myoblasts that acute $(30 \mathrm{~min})$ inhibition of $\mathrm{Cl}$ or $\mathrm{CIII}$ stimulates glycolytic ATP production to prevent ATP shortage. ${ }^{47,48}$ Similarly, chronic ( 5 weeks) $\mathrm{Cl}$ inhibition induced a fully glycolytic phenotype in primary skin fibroblasts, associated with an extreme sensitivity to glucose withdrawal. ${ }^{49}$ These findings led us to propose that BAYinduced $\mathrm{Cl}$ inhibition might, in addition to increasing cellular ROS levels, induce a shortage of glucose contributing to cell death. ${ }^{19}$ Compatible with this idea and our current results, 

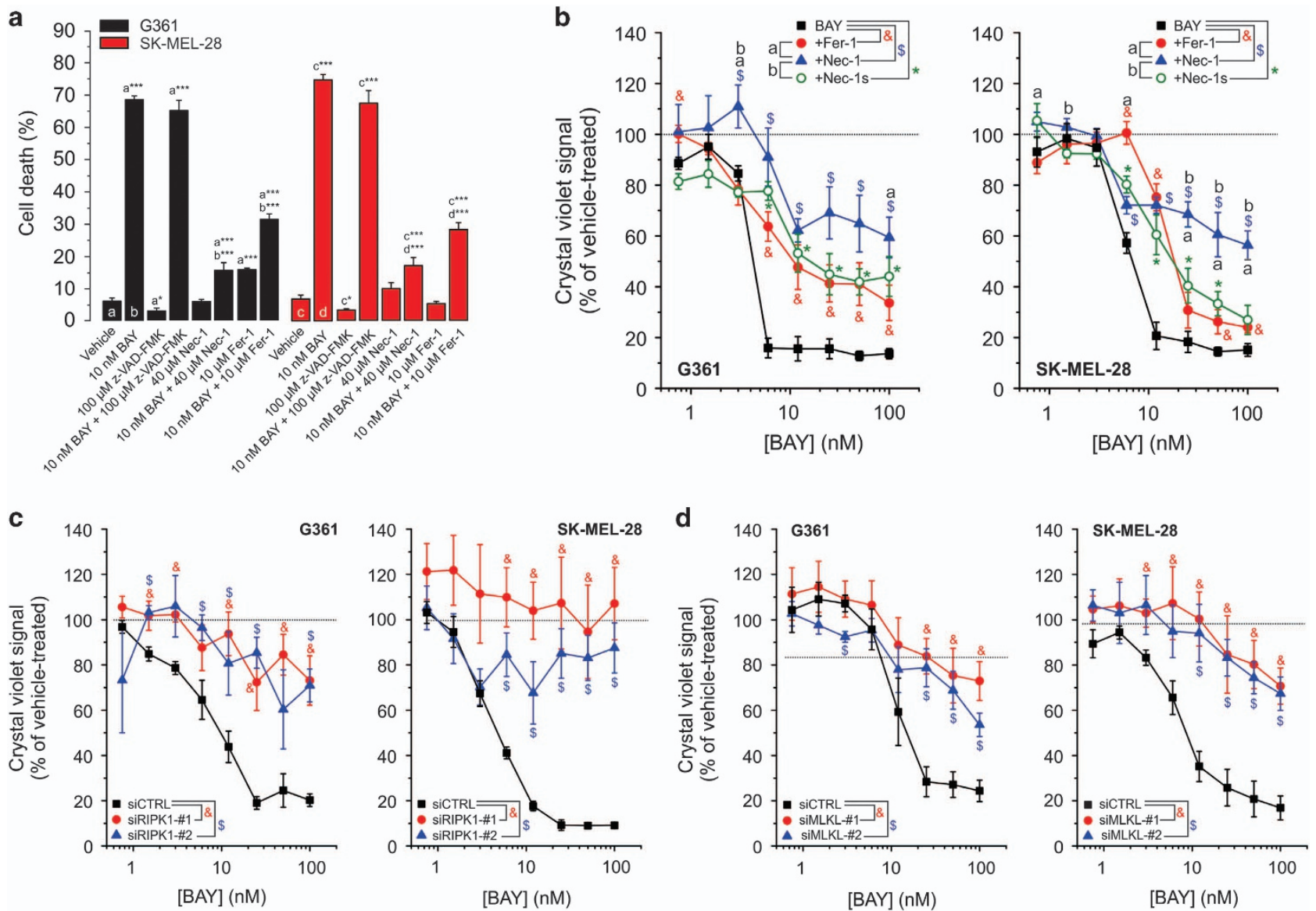

Figure 6 Effect of z-VAD-FMK, Nec-1, Nec-1s, Fer-1 and knockdown of RIPK1 and MLKL on the BAY-induced reduction in cell viability. (a) Effect of vehicle $(N=5, n=15)$, $\operatorname{BAY}(N=5, n=15)$, the pancaspase inhibitor z-VAD-FMK $(N=3, n=9)$, Nec-1 $(N=3, n=9)$ and Fer-1 $(N=3, n=9)$ on cell death (G361: at 48 h; SK-MEL-28: at $72 \mathrm{~h})$. (b) Effect of BAY on viability (G361: at $48 \mathrm{~h}$; SK-MEL-28: at $72 \mathrm{~h} ; \mathrm{N}=3, n=6$ ) in the absence and presence of the ferroptosis inhibitor Fer-1 and the RIPK1 inhibitors Nec-1 and Nec-1s. (c) Effect of BAY on cell viability (G361: at $48 \mathrm{~h}$; SK-MEL-28: at $72 \mathrm{~h} ; N=3, n=6$ ) in cells transfected with siCTRL, siRIPK1-no. 1 and siRIPK1-no. 2. (d) Effect of BAY on the viability of cells (G361: at $48 \mathrm{~h}$; SK-MEL-28: at $72 \mathrm{~h} ; \mathrm{N}=3, n=6$ ) transfected with siCTRL, siMLKI-no. 1 and siMLKL-no. 2. Statistics: Significant differences relative to the marked conditions are indicated by ${ }^{*} P<0.05$ and ${ }^{* * *} P<0.001$. In panels $b-d$, significant differences $(P<0.05)$ between conditions are marked by symbols $(a, b, \&, \$)$

evidence was provided that chronic glucose depletion induces autophagic cell death in B16F1 melanoma cells. ${ }^{50}$ Moreover, we previously observed that increasing the external glucose concentration from 5 to $25 \mathrm{mM}$ inhibited BAY-induced cell death. ${ }^{19}$ Here we demonstrate that regular medium refreshment does not markedly inhibit the BAY-induced reduction in cell viability, arguing against glucose shortage having a role in this process. In this sense, our observation that $25 \mathrm{mM}$ glucose also reduces the BAY-induced ROS increase ${ }^{19}$ suggests that the inhibitory effect of high external glucose on BAY-induced cell death is ROS-mediated.

Role of mPTP opening. Our results highlight an important role for MPTP opening in the death-inducing mechanism of BAY. TRAP1 overexpression also reduced the BAY-induced increase in cellular ROS levels. Supported by evidence in the literature, ${ }^{22,51}$ this suggests that (part of the generated) ROS acts as an upstream activator of MPTP opening (Figure 8: 'triggering ROS'). Inhibition of BAY-induced cell death by TOC might be due to this antioxidant preventing MPTP activation (by scavenging the 'triggering ROS'). Compatible with this explanation, preliminary evidence suggests that the TOC derivative Trolox inhibits MPTP opening in primary human skin fibroblasts (Werner J.H. Koopman, personal observation). Alternatively, TOC might prevent cell death induction by lowering the levels of mitophagy-induced 'killing ROS' (Figure 8). TRAP1 knockdown potentiated the BAY-induced reduction in cell viability, likely caused by increased ROS levels. ${ }^{52}$ This potentiation, in combination with the protective effect of TRAP1 overexpression, suggests that endogenous TRAP1 has a role in protecting the cells against BAY-induced cell death but that these levels and/or TRAP1 activity are insufficiently high. PINK1 can phosphorylate TRAP1 to prevent apoptosis induced by oxidative stress. ${ }^{53}$ This would mean that PINK1 knockdown should reduce TRAP1 activity, thereby potentiating BAY-induced cell death. In contrast, we observed that PINK1 knockdown antagonized the effects of BAY, arguing against PINK1 acting via TRAP1.

Role of autophagosome formation and mitophagy. BAY treatment stimulated autophagosome formation, which was inhibited by the antioxidant TOC. In parallel, TOC also inhibited the BAY-induced increase in cellular ROS levels and lipid peroxidation. This suggests that increased ROS 
a

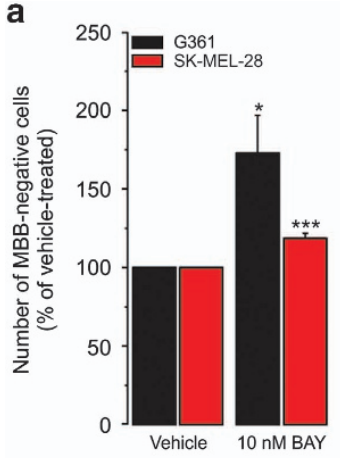

C

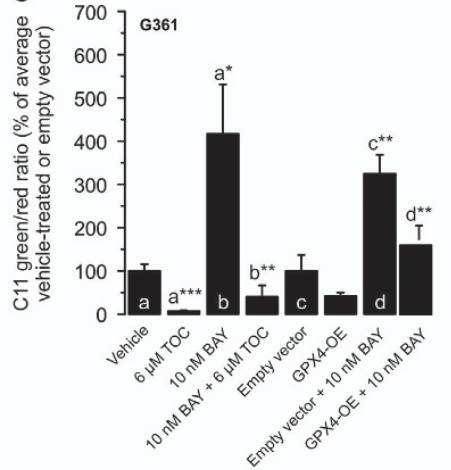

b

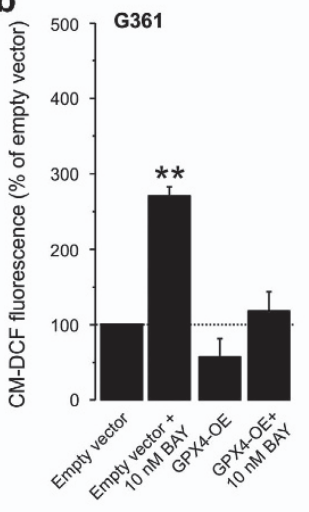

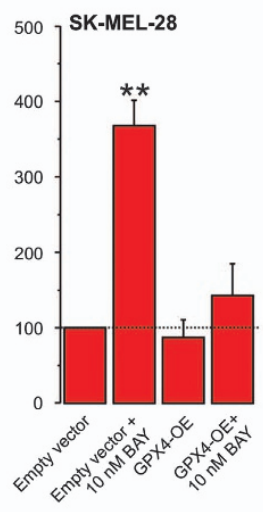

d

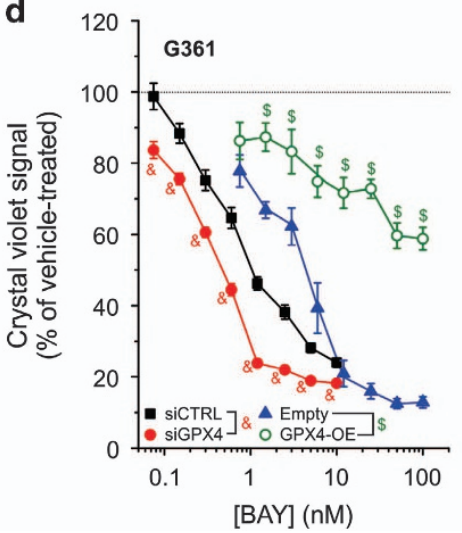

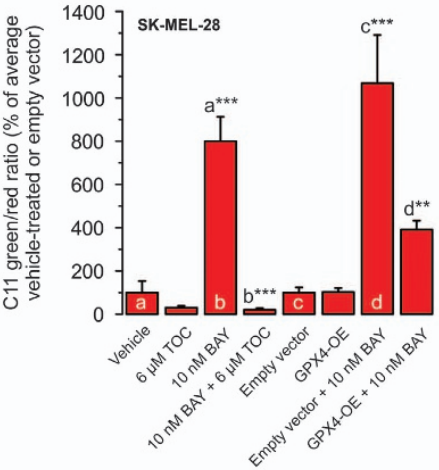

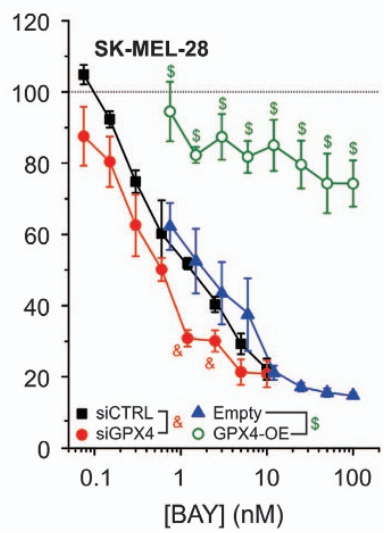

Figure 7 Effect of GPX4 knockdown/overexpression on the BAY-induced reduction in cell viability. (a) Effect of BAY on the number of MBB-negative (GSH-depleted) cells in the vehicle- and BAY-treated condition (G361: at $12 \mathrm{~h}$; SK-MEL-28: at $24 \mathrm{~h} ; N=3, n=9$ ). Higher bars reflect a reduction in the number of MBB-positive cells. (b) Effect of BAY on reactive oxygen species (ROS) levels (at $24 \mathrm{~h} ; N=3, n=3$ ) in cells transfected with the empty or GPX4-OE vector. (c) Cellular lipid peroxidation (at $24 \mathrm{~h}$ ) in vehicle-treated cells (G361: $N=3, n=7$; SK-MEL-28: $N=3, n=6$ ), TOC-treated cells (G361: $N=3, n=7$; SK-MEL-28: $N=3, n=6$ ), BAY-treated cells (G361: $N=3, n=7 ;$ SK-MEL-28: $N=3$, $n=6$ ), BAY+TOC-treated cells (G361: $N=3, n=7$; SK-MEL-28: $N=3, n=6$ ), empty vector-transfected cells (G361: $N=3, n=9 ;$ SK-MEL-28: $N=3, n=8$ ), GPX4-OEtransfected cells (G361: $N=3, n=9$; SK-MEL-28: $N=3, n=8$ ), empty vector-transfected-+BAY-treated cells (G361: $N=3, n=9 ;$ SK-MEL-28: $N=3, n=8$ ) and GPX-OE transfected-+BAY-treated cells (G361: $N=3, n=8$; SK-MEL-28: $N=3, n=8$ ). (d) Effect of BAY on the viability of cells (G361: at $48 \mathrm{~h}$; SK-MEL-28: at $72 \mathrm{~h} ; N=3, n=6$ ) transfected with siCTRL, siGPX4, empty vector and GPX4-OE. Statistics: Significant differences relative to vehicle (panel a), empty vector (panel b) and the marked conditions (panel c) are indicated by ${ }^{*} P<0.05,{ }^{* *} P<0.01,{ }^{*} P<0.01$ and ${ }^{* \star *} P<0.001$. In panel $\mathrm{d}$, significant differences $(P<0.05)$ between conditions are marked by symbols $(\&$, $\$)$

and/or lipid peroxidation are required for stimulation of autophagosome formation, supported by previous findings. ${ }^{28,54-56}$ Upon $\Delta \psi$ depolarization, mitophagy is triggered by PINK1 accumulation on the surface of mitochondria ultimately leading to their autophagosomal uptake and lysosomal degradation. ${ }^{57}$ Knockdown of ATG5 and PINK1 inhibited autophagosome formation and mitophagy, respectively, suggesting that BAY-induced mPTP opening stimulates autophagosome formation, ${ }^{24,26}$ allowing subsequent mitochondrial removal by mitophagy. ${ }^{27}$ Supporting this mechanism is the fact that mitochondrial content was reduced by up to $50 \%$ in BAY-treated cells and that this reduction was inhibited by knockdown of ATG5 or PINK1. Importantly, both ATG5 and PINK1 knockdown prevented the BAY-induced increase in cellular ROS levels and cell viability reduction. Stimulation and inhibition of autophagy in an angiogenesis model also increased and decreased ROS levels, respectively. ${ }^{58}$ This strongly suggests that increased cellular ROS levels and triggering of cell death are downstream effectors of autophagy/mitophagy (Figure 8). Although it might be possible that mitochondria produce increased amounts of ROS at some time during their mitophagic degradation, the exact mechanism by which autophagy/ mitophagy increases ROS levels requires further investigation. PINK1 knockdown/knockout has been associated with mitochondrial fragmentation, increased ROS levels, $\Delta \psi$ depolarization and stimulation of mPTP opening. ${ }^{59-61}$ In contrast, here we observed that PINK1 knockdown by itself induced apparent $\Delta \psi$ hyperpolarization and, consistently, inhibited the BAY-induced stimulation of mitophagy, $\Delta \psi$ depolarization and ROS increase. These effects might be a melanoma- or context-specific phenomenon that, to the best of our knowledge, was not described previously. Whether mitophagy requires prior Drp1-mediated mitochondrial fragmentation is still controversial. ${ }^{62}$ For instance, mitochondrial division during mitophagy can occur in a Drp1independent manner. ${ }^{63}$ On the other hand, Drp1-dependent mitochondrial fragmentation might facilitate mitophagy by creating small-size fragments facilitating autophagosomal uptake. 62

Role of mitochondrial morphology. We demonstrated that mitochondria display a non-filamentous morphology in G361 and SK-MEL-28 cells and that BAY-induced cell death is not 


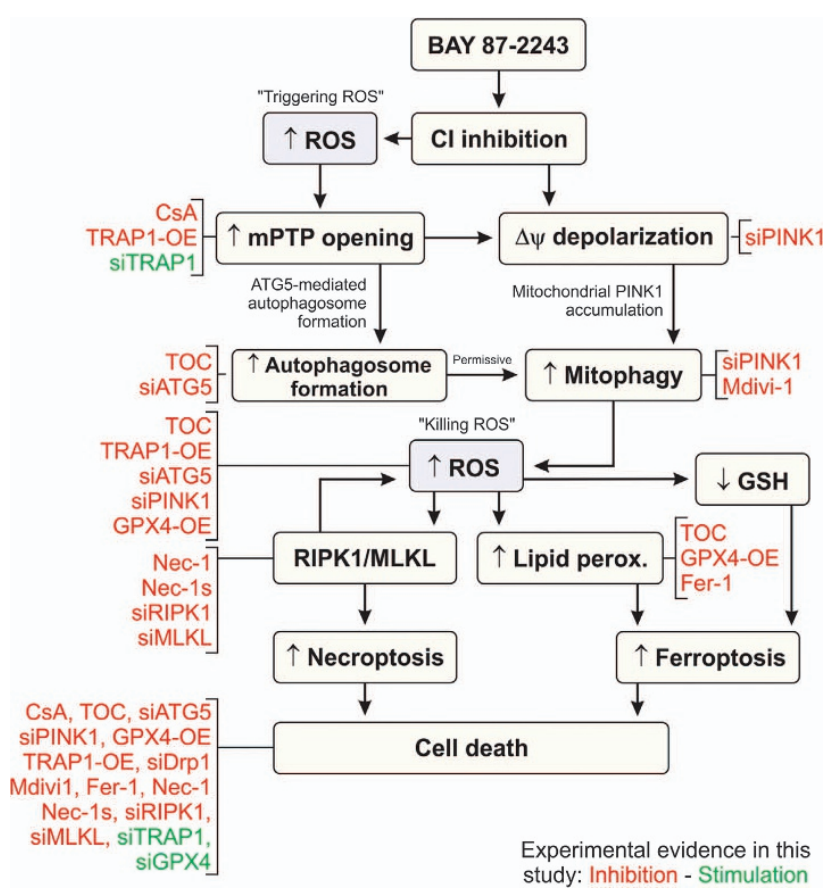

Figure 8 Proposed mechanistic model and experimental evidence. Treatment with $\mathrm{BAY}$ inhibits mitochondrial $\mathrm{Cl}$. This induces a (local) increase in reactive oxygen species (ROS) levels ('triggering ROS'), which stimulates MPTP opening and autophagosome formation. Simultaneously, $\mathrm{Cl}$ inhibition induces depolarization of the mitochondrial membrane potential $(\Delta \psi)$ leading to mitophagy induction. The latter increases ROS levels ('killing ROS') leading to parallel stimulation of necrosome formation (RIPK1/ $M L K L)$, lipid peroxidation and GSH depletion. Increased necrosome formation further stimulates ROS levels and leads to induction of necroptosis, whereas lipid peroxidation and GSH depletion stimulate ferroptosis. Experimental evidence presented in this study are marked in red (inhibitory effect) and green (stimulatory effect)

associated with detectable changes in this morphological phenotype. Drp1 knockdown induced a filamentous mitochondrial morphology that was not affected by BAY treatment and also inhibited the BAY-induced reduction in cell viability. BAYinduced mitophagy and cell death were reduced by Mdivi1, a chemical inhibitor of Drp1 activity. These results suggest that mitochondrial filamentation prevents BAY-induced stimulation of mitophagy and thereby cell death. In parallel, mitochondrial filamentation might also mitigate mitochondrial dysfunction by damage and/or antioxidant sharing, ${ }^{64}$ thereby inhibiting the ROS trigger for MPTP-induced mitophagy induction (Figure 8). In addition to its role in mitophagy PINK1 is a known regulator of the mitochondrial fission and fusion machinery. Using primary neurons and COS-7 cells, it was demonstrated that PINK1 knockdown induces mitochondrial filamentation. ${ }^{65}$ This might suggest that in our study part of the inhibitory effect of PINK1 knockdown on BAY-induced cell death is mediated by stimulation of mitochondrial filamentation. Interestingly, melanoma cells that were made resistant against the BRAF ${ }^{\mathrm{V} 600 \mathrm{E}_{\text {. }}}$ specific inhibitor and chemotherapy drug Vemurafenib possessed filamentous mitochondria. ${ }^{66}$ Conversely, knockdown of the fusion-promoting protein mitofusin 2 in melanoma cells induced a more fragmented mitochondrial phenotype and increased cell death upon Vemurafenib treatment. ${ }^{67}$ Therefore, our results might suggest that prevention or reversal of mitochondrial filamentation could be a strategy to overcome Vemurafenib resistance in $\mathrm{BRAF}^{\mathrm{V} 600 \mathrm{E}}$ melanoma cells.

Mode of cell death. We observed that z-VAD-FMK neither displayed cytotoxic effects by itself nor prevented BAYinduced cell death, arguing against involvement of apoptosis. The inhibitory effects of Nec-1s and Fer-1 suggest that combined necroptosis and ferroptosis are responsible for the BAY-induced reduction in cell viability. Involvement of necroptosis is further supported by the inhibitory effects of RIPK1 and MLKL knockdown on the BAY-induced reduction in cell viability. Compatible with the proposed mechanism (Figure 8), increased ROS levels have been demonstrated to promote stabilization of the RIPK1/RIPK3 necrosome. ${ }^{68}$ The latter study also provided evidence that RIPK1, RIPK3 and MLKL stimulate ROS production, which further promotes necrosome stabilization. Involvement of ferroptosis is supported by the observation that BAY treatment increases ROS levels, stimulates lipid peroxidation and induces GSH depletion, all of which are hallmarks of ferroptotic cell death. ${ }^{41}$ It was recently demonstrated that oxidation of specific phosphatidylethanolamines (PEs) in endoplasmicreticulum-associated cell compartments depends on acylCoA synthase 4 and is required for ferroptosis induction. ${ }^{69,70}$ Compatible with our TOC results, tocopherols suppressed ferroptosis by inhibiting lipoxygenase, which generates doubly and triply oxygenated (15-hydroperoxy)-diacylated PE species that act as death signals. ${ }^{69,70}$ Knockdown and overexpression of GPX4, an essential regulator of ferroptotic cell death, ${ }^{69-71}$ potentiated and antagonized the BAY-induced reduction in cell viability, respectively. This again suggests involvement of ferroptosis in BAY-induced cell death. It is currently unclear how increased cellular ROS levels induce specific activation of necroptosis or ferroptosis. ${ }^{72} \mathrm{Nec}-1 \mathrm{~s}$ and Fer-1 inhibited the BAY-induced reduction in cell viability to a similar extent, suggesting that both death mechanisms are equally activated. Erastin-induced ferroptosis was promoted by autophagic degradation of ferritin and ATG5 knockdown inhibited this ferroptosis. ${ }^{44}$ Therefore, BAY-induced stimulation of autophagy might directly activate ferroptosis. However, knockdown of ATG5 or PINK1 prevented the BAY-induced reduction in cell viability to a similar extent, suggesting that PINK1-dependent mitophagy, occurring downstream of ATG5-dependent autophagosome formation, is primarily responsible for cell death induction.

\section{Conclusion}

Using BAY as a tool, we here provide evidence that $\mathrm{Cl}$ inhibition induces the death of BRAF ${ }^{\mathrm{V} 600 \mathrm{E}}$ melanoma cells by stimulating MPTP opening and inducing $\Delta \psi$ depolarization. These events increase cellular ROS levels in a mitophagydependent manner, leading to induction of combined necroptotic/ferroptotic cell death.

\section{Materials and Methods}

Cell culture. BRAF ${ }^{\mathrm{V} 600 E}$ human melanoma cell lines G361 (no. ATCCCRL-1424) and SK-MEL-28 (no. ATCC-HTB-72) were derived from a skin melanoma site and obtained from American Type Culture Collection (ATCC; LGC Standards $\mathrm{GmbH}$, Wesel, Germany). Melanoma cells were routinely cultured at 
$37{ }^{\circ} \mathrm{C}$ and $5 \% \mathrm{CO}_{2}$ in ATCC-recommended media supplemented with $10 \%$ (v/v) fetal calf serum (FCS; Gibco-Thermo-Fisher, Waltham, MA, USA). Before experiments, the above cells were cultured for $12 \mathrm{~h}$ in a pyruvate-free medium (no. A1443001; Gibco Thermo Fisher Scientific Inc., Waltham, MA, USA) to which was added: $5 \mathrm{mM} \mathrm{D}$ glucose (Sigma-Aldrich, St. Louis, MO, USA), 2 mM GlutaMAX (no. 35050038; Gibco) and 5\% (v/v) dialyzed FCS (no. 26400044; 10000 MW cutoff; Gibco). Cells were routinely cultured in a humidified atmosphere $\left(95 \%\right.$ air, $\left.5 \% \mathrm{CO}_{2}, 37^{\circ} \mathrm{C}\right)$.

Knockdown/overexpression studies and western blot analysis. Knockdown/overexpression was performed as described in the Supplementary Information. For western blotting, cell pellets were lysed in modified RIPA buffer (150 mM sodium chloride, $1 \%$ Triton X-100, 0.5\% sodium deoxycholate, $0.1 \%$ SDS, $50 \mathrm{mM}$ Tris, pH 8.0) containing 1x protease inhibitor cocktail (no. 116974898001; Roche, Mannheim, Germany). Protein concentration in the lysates was determined using a NanoDrop UV-Vis Spectrophotometer (Thermo Fisher Scientific Inc. Wilmington, DE, USA), and $100 \mu \mathrm{g}$ of protein per well was separated by SDS-PAGE and transferred to a PDVF membrane using an iBlot gel transfer stack (Invitrogen, Carlsbad, CA, USA). Antibodies and their detection are described in the Supplementary Information.

Cell viability, death assay and MPTP opening. Cell viability was determined using crystal violet staining. Cell death was assessed by flowcytometric analysis of propidium iodide (PI)-stained cells using a FACSCalibur flow cytometer (BD Biosciences, Breda, The Netherlands). Analysis of mPTP opening was performed using a previously described approach. ${ }^{22}$ Additional information is provided in the Supplementary Information.

Quantification of autophagy, mitophagy and mitochondrial morphology. For autophagy analysis, cells were seeded on Nunc Lab-Tek glass-bottomed coverslips (no. 565470; Thermo Scientific), transfected with a construct encoding tandem mCherry-GFP-tagged LC3 (no. 21074; Addgene, Cambridge, MA, USA) and cultured for $24 \mathrm{~h}$. Mitophagy was analyzed by transfecting the cells with a GFP-LC3 construct (no. P36235; Invitrogen), followed by subsequent costaining with MR (Invitrogen). Mitochondrial morphology was analyzed by staining the cells with MG (Invitrogen). Details are provided in the Supplementary Information.

Analyses of cellular ROS levels, GSH levels, lipid peroxidation, mitochondrial membrane potential and mitochondrial content. To measure ROS and GSH levels, cells were stained with CM- $\mathrm{H}_{2}$ DCFDA or monobromobimane (MBB), respectively. Cellular lipid peroxidation was quantified using C11-BODIPY ('C $\mathrm{C} 11^{\prime}$ ). Mitochondrial membrane potential was quantified using $5,5^{\prime}, 6,6^{\prime}$-tetrachloro-1, 1',3,3'-tetraethylbenzimidazolyl-carbocyanine iodide ('JC-1') staining. Mitochondrial content was determined using MG staining. Details are provided in the Supplementary Information.

Chemicals. N-benzyloxycarbonyl-Val-Ala-Asp-fluoromethylketone (no. ALX260020; Z-VAD-FMK) was obtained from Enzo Life Sciences (Raamsdonkveer, The Netherlands) and Nec-1 (no. SC200142) from Santa Cruz Biotech (Dallas, TX USA). (+)-TOC (no. T3251), BafA1 (no. B1793), Crystal Violet solution (no. HT90132), CsA (no. 30024), Fer-1 (no. SML0583), Mdivi1 (no. M0199) and PI (no. P4170; Pl) were obtained from from Sigma-Aldrich. Nec-1s (no. HY-14622A) was purchased from Bio-Connect BV (Huissen, The Netherlands). BAY) was provided by Bayer AG (Leverkusen, Germany).

Data and image analysis. The number of independent experiments (days) and replicates (assays, individual cells) are marked by $N$ and $n$, respectively. Unless stated otherwise, statistical significance was assessed using an independent two-population Student's $t$-test $\left({ }^{\star} P<0.05 ;{ }^{* \star} P<0.01\right.$; ${ }^{* \star} P<0.001$; relative to the indicated condition), and results from multiple experiments are represented by their average value \pm S.E.M. Curve fitting and statistical analysis was performed using Origin Pro 6.1 (OriginLab, Northampton, MA, USA). Image visualization, processing and quantification was carried out using Image Pro Plus 6.1 (Media Cybernetics, Rockville, MD, USA) and FIJI software (HTTP://fiji.sc/).

\section{Conflict of Interest}

FB, LMPEVO, HMB, SEvEdV, JCWH, SG, PHGMW and WJHK declare no competing interests. LS, CK and MH are full-time employees of Bayer Pharma AG, which developed BAY 87-2243.
Acknowledgements. This research was supported by the Nimbus Initiative (Bayer AG), the Radboud Nanomedicine Alliance and an equipment grant of NWO (Netherlands Organization for Scientific Research, No: 911-02-008). We are grateful to Prof. Dr. M Conrad (Helmholtz Zentrum München, Munich, Germany) for providing the GPX4-pcDNA3 construct.

\section{Author contributions}

FB: designed experiments, performed experiments, analyzed data, prepared figures, wrote the manuscript; LMPEVO: performed experiments, analyzed data; LS: designed experiments, discussed and proofread the manuscript; HMB: performed experiments, analyzed data; SEvEdV: performed experiments; JCWH: performed experiments, analyzed data; SG: designed experiments, performed experiments, analyzed data; CK: discussed and proofread the manuscript; MH: discussed and proofread the manuscript; PHGMW: designed experiments, wrote the manuscript; WJHK: designed experiments, analyzed data, prepared figures, wrote the manuscript, supervised the research.

1. Abildgaard C, Guldberg P. Molecular drivers of cellular metabolic reprogramming in melanoma. TR Mol Med 2015; 21: 164-171.

2. Yuneva MO, Fan TW, Allen TD, Higashi RM, Ferraris DV, Tsukamoto T et al. The metabolic profile of tumors depends on both the responsible genetic lesion and tissue type. Cell Metab 2012; 15: 157-170.

3. Shakya A, Cooksey R, Cox JE, Wang V, McClain DA, Tantin D. Oct1 loss of function induces a coordinate metabolic shift that opposes tumorigenicity. Nat Cell Biol 2009; 11: 320-327.

4. Gough DJ, Corlett A, Schlessinger K, Wegrzyn J, Larner AC, Levy DE. Mitochondrial STAT3 supports Ras-dependent oncogenic transformation. Science 2009; 324: 1713-1716.

5. Rossignol R, Gilkerson R, Aggeler R, Yamagata K, Remington SJ, Capaldi RA. Energy substrate modulates mitochondrial structure and oxidative capacity in cancer cells. Cancer Res 2004; 64: 985-993.

6. Garnett MJ, Marais R. Guilty as charged: B-RAF is a human oncogene. Cancer Cell 2004; 6: 313-319.

7. Davies H, Bignell GR, Cox C, Stephens P, Edkins S, Clegg S et al. Mutations of the BRAF gene in human cancer. Nature 2002; 417: 949-954.

8. Sullivan RJ, Flaherty KT. Resistance to BRAF-targeted therapy in melanoma. Eur J Cancer 2013; 49: 1297-1304.

9. Gopal YN, Rizos H, Chen G, Deng W, Frederick DT, Cooper ZA et al. Inhibition of mTORC1/2 overcomes resistance to MAPK pathway inhibitors mediated by PGC1 $\alpha$ and oxidative phosphorylation in melanoma. Cancer Res 2014; 74: 7037-7047.

10. Haq R, Shoag J, Andreu-Perez P, Yokoyama S, Edelman H, Rowe GC et al. Oncogenic BRAF regulates oxidative metabolism via PGC1 $\alpha$ and MITF. Cancer Cell 2013; 23: 302-315.

11. Weinberg SE, Chandel NS. Targeting mitochondria metabolism for cancer therapy. Nat Chem Biol 2015; 11: 9-15.

12. Vazquez F, Lim JH, Chim H, Bhalla K, Gimun G, Pierce K et al. PGC1 $\alpha$ expression defines a subset of human melanoma tumors with increased mitochondrial capacity and resistance to oxidative stress. Cancer Cell 2013; 23: 287-301.

13. Gorman GS, Chinnery PF, DiMauro S, Hirano M, Koga Y, McFarland R et al. Mitochondrial diseases. Nat Rev Dis Primers 2016; 2: 16080.

14. Koopman WJH, Beyrath J, Fung CW, Koene S, Rodenburg RJ, Willems PH et al. Mitochondrial disorders in children: toward development of small-molecule treatment strategies. EMBO Mol Med 2016; 8: 311-327.

15. Vafai SB, Mootha VK. Mitochondrial disorders as windows into an ancient organelle. Nature 2012; 491: 374-383.

16. Mitchell $P$. Coupling of phosphorylation to electron and hydrogen transfer by a chemi-osmotic type of mechanism. Nature 1961; 191: 144-148.

17. Koopman WJH, Distelmaier F, Esseling JJ, Smeitink JAM, Willems PH. Computer-assisted live cell analysis of mitochondrial membrane potential, morphology and calcium handling. Methods 2008; 46: 304-311.

18. Ellinghaus $\mathrm{P}$, Heisler I, Unterschemmann $\mathrm{K}$, Haerter M, Beck $\mathrm{H}$, Greschat $\mathrm{S}$ et al. BAY $87-2243$, a highly potent and selective inhibitor of hypoxia-induced gene activation has antitumor activities by inhibition of mitochondrial complex I. Cancer Med 2013; 2: 611-624.

19. Schöckel L, Glasauer A, Basit F, Bitschar K, Truong H, Erdmann G et al. Targeting mitochondrial complex I using BAY 87-2243 reduces melanoma tumor growth. Cancer Metab 2015; 3: 11.

20. Condelli V, Maddalena F, Sisinni L, Lettini G, Matassa DS, Piscazzi A et al. Targeting TRAP1 as a downstream effector of BRAF cytoprotective pathway: a novel strategy for human BRAF-driven colorectal carcinoma. Oncotarget 2015; 6: 22298-22309.

21. Im CN. Past, present, and emerging roles of mitochondrial heat shock protein TRAP1 in the metabolism and regulation of cancer stem cells. Cell Stress Chaperones 2016; 21: 553-562.

22. Blanchet L, Grefte S, Smeitink JA, Willems PH, Koopman WJ. Photo-induction and automated quantification of reversible mitochondrial permeability transition pore opening in primary mouse myotubes. PLOS ONE 2014; 9: e114090.

23. lannetti EF, Smeitink JA, Beyrath J, Willems PH, Koopman WJ. Multiplexed high-content analysis of mitochondrial morphofunction using live-cell microscopy. Nat Protoc 2016; 11: 1693-1710. 
24. Sica V, Galluzzi L, Bravo-San Pedro JM, Izzo V, Maiuri MC, Kroemer G. Organelle-specific initiation of autophagy. Mol Cell 2015; 59: 522-539.

25. Klionsky DJ. The autophagosome is overrated!. Autophagy 2011; 7: 353-354

26. Nakatogawa H, Suzuki K, Kamada Y, Ohsumi Y. Dynamics and diversity in autophagy mechanisms: lessons from yeast. Nat Rev Mol Cell Biol 2009; 10: 458-467.

27. Lazarou M, Sliter DA, Kane LA, Sarraf SA, Wang C, Burman JL et al. The ubiquitin kinase PINK1 recruits autophagy receptors to induce mitophagy. Nature 2015; 524: 309-314.

28. Twig G, Elorza A, Molina AJ, Mohamed H, Wikstrom JD, Waltzer G et al. Fission and selective fusion govern mitochondrial segregation and elimination by autophagy. EMBO $J$ 2008; 27: 433-446.

29. Rambold AS, Kostelecky B, Elia N, Lippincott-Schwartz J. Tubular network formation protects mitochondria from autophagosomal degradation during nutrient starvation. Proc Natl Acad Sci USA 2011; 108: 10190-10195.

30. Willems PH, Rossignol R, Dieteren CE, Murphy MP, Koopman WJH. Redox homeostasis and mitochondrial dynamics. Cell Metab 2015; 22: 207-218.

31. Cassidy-Stone A, Chipuk JE, Ingerman E, Song C, Yoo C, Kuwana T et al. Chemical inhibition of the mitochondrial division dynamin reveals its role in Bax/Bak-dependent mitochondrial outer membrane permeabilization. Dev Cell 2008; 14: 193-204.

32. Goodall ML, Fitzwalter BE, Zahedi S, Wu M, Rodriguez D, Mulcahy-Levy JM et al. The autophagy machinery controls cell death switching between apoptosis and necroptosis. Dev Cell 2016; 37: 337-349.

33. Fitzwalter BE, Thorburn A. Recent insights into cell death and autophagy. FEBS J 2015; 282 $4279-4288$.

34. Basit F, Cristofanon S, Fulda S. Obatoclax (GX15-070) triggers necroptosis by promoting the assembly of the necrosome on autophagosomal membranes. Cell Death Differ 2013; 20: 1161-1173.

35. Sentelle RD, Senkal CE, Jiang W, Ponnusamy S, Gencer S, Selvam SP et al. Ceramide targets autophagosomes to mitochondria and induces lethal mitophagy. Nat Chem Biol 2012; 8: 831-838.

36. Yousefi S, Perozzo R, Schmid I, Ziemiecki A, Schaffner T, Scapozza L et al. Calpain-mediated cleavage of Atg5 switches autophagy to apoptosis. Nat Cell Biol 2006; 8: 1124-1132.

37. Conrad M, Angeli JP, Vandenabeele P, Stockwell BR. Regulated necrosis: disease relevance and therapeutic opportunities. Nat Rev Drug Discov 2016; 15: 348-366.

38. Degterev A, Huang Z, Boyce M, Li Y, Jagtap P, Mizushima N et al. Chemical inhibitor of nonapoptotic cell death with therapeutic potential for ischemic brain injury. Nat Chem Biol 2005; 1: 112-119.

39. Vandenabeele P, Grootjans S, Callewaert N, Takahashi N. Necrostatin-1 blocks both RIPK1 and IDO: consequences for the study of cell death in experimental disease models. Cell Death Diff 2013; 20: 185-187.

40. Muller AJ, DuHadaway JB, Donover PS, Sutanto-Ward E, Prendergast GC. Inhibition of indoleamine 2,3-dioxygenase, an immunoregulatory target of the cancer suppression gene Bin1, potentiates cancer chemotherapy. Nat Med 2005; 11: 312-319.

41. Galluzzi L, Kepp O, Chan FK, Kroemer G. Necroptosis: mechanisms and relevance to disease. Annu Rev Pathol Mech Dis 2017; 12: 103-130.

42. Remijsen Q, Goossens V, Grootjans S, Van den Haute C, Vanlangenakker N, Dondelinger $Y$ et al. Depletion of RIPK3 or MLKL blocks TNF-driven necroptosis and switches towards a delayed RIPK1 kinase-dependent apoptosis. Cell Death Dis 2014; 5: e1004.

43. Wallach D, Kang TB, Dillon CP, Green DR. Programmed necrosis in inflammation: toward identification of the effector molecules. Science 2016; 352: aaf2154.

44. Hou W, Xie Y, Song X, Sun X, Lotze MT, Zeh HJ 3rd et al. Autophagy promotes ferroptosis by degradation of ferritin. Autophagy 2016; 12: 1425-1428.

45. Dixon SJ, Lemberg KM, Lamprecht MR, Skouta R, Zaitsev EM, Gleason CE et al Ferroptosis: an iron-dependent form of nonapoptotic cell death. Cell 2012; 149: 1060-1072.

46. Galadari S, Rahman A, Pallichankandy S, Thayyullathil F. Reactive oxygen species and cancer paradox: to promote or to suppress? Free Radic Biol Med 2017; 104: 144-164

47. Liemburg-Apers DC, Schirris TJ, Russel FG, Willems PH, Koopman WJH. Mitoenergetic dysfunction triggers a rapid compensatory increase in steady-state glucose flux. Biophys J 2015; 109: 1372-1386.

48. Liemburg-Apers DC, Wagenaars JAL, Smeitink JAM, Willems PH, Koopman WJH. Acute stimulation of glucose influx upon mitoenergetic dysfunction requires LKB1, AMPK, Sirt2 and mTOR-RAPTOR. J Cell Sci 2016; 129: 4411-4423.

49. Distelmaier F, Valsecchi F, Liemburg-Apers DC, Lebiedzinska M, Rodenburg RJ, Heil S et al. Mitochondrial dysfunction in primary human fibroblasts triggers an adaptive cell survival program that requires AMPK- $\alpha$. Biochim Biophys Acta 2015; 1852: 529-540.

50. Chen Y, Liersch R, Detmar M. The miR-290-295 cluster suppresses autophagic cell death of melanoma cells. Sci Rep 2012; 2: 808.

51. Izzo V, Bravo-San Pedro JM, Sica V, Kroemer G, Galluzzi L. Mitochondrial permeability transition: new findings and persisting uncertainties. TR Cell Biol 2016; 26: 655-667.
52. Hua $G$, Zhang $Q$, Fan $Z$. Heat shock protein 75 (TRAP1) antagonizes reactive oxygen species generation and protects cells from granzyme M-mediated apoptosis. J Biol Chem 2007; 282: 20553-20560.

53. Pridgeon JW, Olzmann JA, Chin LS, Li L. PINK1 protects against oxidative stress by phosphorylating mitochondrial chaperone TRAP1. PLOS Biol 2007; 5: e172.

54. Pryde KR, Smith HL, Chau KY, Schapira AH. PINK1 disables the anti-fission machinery to segregate damaged mitochondria for mitophagy. J Cell Biol 2016; 213: 163-171.

55. Song M, Mihara K, Chen Y, Scorrano L, Dorn GW II. Mitochondrial fission and fusion factors reciprocally orchestrate mitophagic culling in mouse hearts and cultured fibroblasts. Cell Metab 2015; 21: 273-285.

56. Frank M, Duvezin-Caubet S, Koob S, Occhipinti A, Jagasia R, Petcherski A et al. Mitophagy is triggered by mild oxidative stress in a mitochondrial fission dependent manner. Biochim Biophys Acta 2012; 1823: 2297-2310.

57. Nguyen TN, Padman BS, Lazarou M. Deciphering the molecular signals of PINK1/Parkin mitophagy. TR Cell Biol 2016; 26: 733-744

58. Du J, Teng RJ, Guan T, Eis A, Kaul S, Konduri GG et al. Role of autophagy in angiogenesis in aortic endothelial cells. Am J Physiol Cell Physiol 2012; 302: C383-C391.

59. Gautier CA, Giame E, Caballero E, Núnez L, Song Z, Chan D et al. Regulation of mitochondrial transition pore by PINK1. Mol Neurodegener 2012; 7: 22.

60. Abramov AY, Gegg M, Grunewald A, Wood NW, Klein C, Schapira AH. Bioenergetic consequences of PINK1 mutations in Parkinson disease. PLOS ONE 2011; 6: e25662.

61. Heeman B, Van den Haute C, Aelvoet SA, Valsecchi F, Rodenburg RJ, Reumers V et al. Depletion of PINK1 affects mitochondrial metabolism, calcium homeostasis and energy maintenance. J Cell Sci 2011; 124: 1115-1125.

62. Graef M. A dividing matter: Drp1/Dnm1-independent mitophagy. J Cell Biol 2016; 215 599-601.

63. Yamashita SI, Jin X, Furukawa K, Hamasaki M, Nezu A, Otera H et al. Mitochondrial division occurs concurrently with autophagosome formation but independently of Drp1 during mitophagy. J Cell Biol 2016; 215: 649-665.

64. Neuspiel M, Zunino R, Gangaraju S, Rippstein P, McBride H. Activated mitofusin 2 signals mitochondrial fusion, interferes with Bax activation, and reduces susceptibility to radical induced depolarization. J Biol Chem 2005; 280: 25060-25070.

65. Yang $Y$, Ouyang $Y$, Yang L, Beal MF, McQuibban A, Vogel $H$ et al. Pink1 regulates mitochondrial dynamics through interaction with the fission/fusion machinery. Proc Natl Acad Sci USA 2008; 105: 7070-7075.

66. Baenke F, Chaneton B, Smith M, Van Den Broek N, Hogan K, Tang H et al. Resistance to BRAF inhibitors induces glutamine dependency in melanoma cells. Mol Oncol 2016; 10 : 73-84.

67. Corazao-Rozas P, Guerreschi P, Andre F, Gabert PE, Lancel S, Dekiouk $S$ et al. Mitochondrial oxidative phosphorylation controls cancer cell's life and death decisions upon exposure to MAPK inhibitors. Oncotarget 2016; 7: 39473-39485.

68. Schenk B, Fulda S. Reactive oxygen species regulate Smac mimetic/TNF $\alpha$-induced necroptotic signaling and cell death. Oncogene 2015; 34: 5796-5806.

69. Doll S, Proneth B, Tyurina YY, Panzilius E, Kobayashi S, Ingold I et al. ACSL4 dictates ferroptosis sensitivity by shaping cellular lipid composition. Nat Chem Biol 2017; 13: 91-98.

70. Kagan VE, Mao G, Qu F, Angeli JP, Doll S, Croix CS et al. Oxidized arachidonic acid and adrenic Pes navigate cells to ferroptosis. Nat Chem Biol 2017; 13: 81-90.

71. Yang WS, SriRamaratnam R, Welsch ME, Shimada K, Skouta R, Viswanathan VS et al. Regulation of ferroptotic cancer cell death by GPX4. Cell 2014; 156: 317-331.

72. Xie $Y$, Hou W, Song X, Yu Y, Huang J, Sun X et al. Ferroptosis: process and function Cell Death Differ 2016; 23: 369-379.

Cell Death and Disease is an open-access journal published by Nature Publishing Group. This work is licensed under a Creative Commons Attribution 4.0 International License. The images or other third party material in this article are included in the article's Creative Commons license, unless indicated otherwise in the credit line; if the material is not included under the Creative Commons license, users will need to obtain permission from the license holder to reproduce the material. To view a copy of this license, visit http://creativecommons.org/licenses/by/4.0/

(C) The Author(s) 2017 